\title{
DFD-01 Reduces Transepidermal Water Loss and Improves Skin Hydration and Flexibility
}

\author{
J. Mark Jackson · Gary L. Grove $\cdot$ Kent Allenby $\cdot$ Tim Houser
}

Received: August 3, 2017 / Published online: November 13, 2017

(C) The Author(s) 2017. This article is an open access publication

\section{ABSTRACT}

Introduction: In plaque psoriasis, the benefit of topical steroids is well established. The vehicle formulation of topical steroids may also provide benefit in addition to the effects of the steroid itself. DFD-01 (betamethasone dipropionate spray, $0.05 \%$ ) is a formulation composed of a topical steroid in an emollient-like vehicle that enhances penetration to the target site of inflammation in the skin. The aim of this study was to assess the effect of DFD-01 and its vehicle on skin hydration and barrier function in compromised skin and to evaluate its effect on flexibility in healthy skin.

Methods: Eighteen healthy white volunteers were enrolled in each of two studies. In Study 1,

Enhanced content To view enhanced content for this article go to http://www.medengine.com/Redeem/ ODCCF0603B9791DE.

J. M. Jackson

Division of Dermatology, University of Louisville,

Louisville, KY, USA

G. L. Grove · T. Houser

cyberDERM Clinical Studies, Broomall, PA, USA

K. Allenby $(\bowtie)$

Promius Pharma/Dr. Reddy's Laboratories,

Princeton, NJ, USA

e-mail: abennett@promiuspharma.com dry shaving of volar forearms created a compromised skin barrier, through which transepidermal water loss (TEWL) was measured using an evaporimeter. Capacitance, a measure of epidermal hydration, was also measured at baseline and at 1,2 and $4 \mathrm{~h}$ after application of DFD-01 or its vehicle formulation. In Study 2, intact skin flexibility was tested with a cutometer before and at 1,2 and $4 \mathrm{~h}$ after application of DFD-01 or vehicle.

Results: In Study 1, both DFD-01 and its vehicle were effective at reducing TEWL through the compromised stratum corneum. Capacitance measurements confirmed this finding; razor-chafed skin treated with either DFD-01 or vehicle exhibited levels of skin hydration similar to unshaved control skin. Study 2 found softening and greater flexibility of normal skin treated with either DFD-01 or vehicle compared with nontreated control skin samples.

Conclusions: These tests suggest that the DFD-01 formulation and its vehicle are each effective at retaining moisture within a damaged skin barrier and for softening and increasing the flexibility of intact skin.

Funding: Dr. Reddy's Laboratories.

Keywords: Betamethasone dipropionate spray; Emollient; Formulation; Moisturization; Psoriasis; Skin barrier; Skin flexibility; Transepidermal water loss; Topical steroids 


\section{INTRODUCTION}

There is a sharp contrast between healthy, hydrated skin and eczematous or psoriatic skin that is dry and scaly and has poor barrier function [1]. In plaque psoriasis, the well-demarcated plaques, with their characteristic overlying silvery scale and erythema, may be painful, itchy and uncomfortable [2]. About $85 \%$ of patients with psoriasis suffer from pruritus, which can be severe and may interfere with sleep [3] and the ability to concentrate [4]. Psoriasis may be exacerbated by stress, infections and skin drying [5].

Emollients are moisturizers that help skin feel more comfortable and less itchy by keeping it moist and intact $[6,7]$. Emollients can restore and protect skin barrier function, increase moisture retention in the skin, increase remission times between flares and enhance symptom control in patients with psoriasis [8].

Topical steroids are the mainstay of treatment for the majority of patients with psoriasis with limited disease $(<5 \%$ body surface area) [9]. They are also used for patients with more extensive disease in conjunction with other therapies, including systemic or biologic medications or ultraviolet light therapy [9]. When used as recommended, topical steroids provide efficacy with a good safety profile for the majority of patients.

An agent that provides the combination of emollient-like vehicle and steroid can address skin discomfort and itching, as well as the underlying immunologic disturbance of psoriasis. DFD-01 [Sernivo ${ }^{\mathrm{TM}}$ (betamethasone dipropionate) Spray, 0.05\%], Promius Pharma, Princeton, NJ) is a medium-potency steroid formulation that has demonstrated efficacy in the treatment of extensive, moderate psoriasis, including more difficult-to-treat areas such as knees and elbows $[10,11]$.

This article describes two studies conducted on healthy skin to assess the effect of DFD-01 and its vehicle (DFD-01 without steroid) on skin hydration, barrier function and flexibility. Enhanced skin flexibility, improved epidermal hydration and reduced water loss are indicative of an emollient-like effect, which may have the potential to ease the skin discomfort associated with psoriasis.

\section{METHODS}

\section{Study 1: Transepidermal Water Loss}

Enrolled subjects were healthy white males and females, aged 18-55 years, with Fitzpatrick skin type I, II or III. Subjects were excluded if they had any of the following: type I or II diabetes; current tobacco use; any skin condition on the volar forearms other than dry skin (e.g., psoriasis, eczema, atopic dermatitis); marks, scars, moles, scratches or other blemishes on the volar forearm test sites that would interfere with the study; or known allergies or sensitivities to any topical corticosteroid product or cosmetics, soaps or fragrances.

Participants visited the test site on 2 consecutive days, each day wearing a short-sleeved shirt and having not showered within the hour prior to their visits. Participants were required to discontinue use of all moisturizing products (e.g., soaps, lotions, sunscreens, insect repellents, etc.) on the volar forearms for the 3 days prior to the start of the study and for the duration of the study.

On day 1 , volunteers were dry shaved with a disposable razor to create four 5 by $5-\mathrm{cm}$ test sites on both volar forearms (two sites per forearm). Dry shaving disrupts the integrity of the stratum corneum and creates a damaged skin barrier. Evaporimeter measurements (RG1 Evaporimeter system, cyberDERM, Broomall, PA) with transepidermal water loss (TEWL) probes (Cortex Technology, Hadsund, Denmark) were conducted after shaving to confirm elevated water loss and provide a measure of skin barrier function. Both probes of the Evaporimeter contain sensors that measure the temperature and relative humidity at two fixed points along the axis perpendicular to the skin surface. Water loss data were collected at a rate of four inputs/s over a 20-s interval once steady-state conditions had been achieved to provide an average of evaporative water loss rate in $\mathrm{gm} / \mathrm{m}^{2} / \mathrm{h}$.

On day 2, the four test sites were evaluated with the evaporimeter for post-shaving TEWL. Measurements were taken following a minimum 25-min acclimatization period in a 
controlled environment with the relative humidity maintained at $<50 \%$ and temperature maintained between 19 and $22^{\circ} \mathrm{C}$. Capacitance, which measures epidermal hydration, was recorded at the same time as TEWL with a Corneometer $^{\circledR}$ CM 825 (Courage + Khazaka Electronic GmbH, Cologne, Germany). Following these baseline readings, DFD-01 or vehicle $(0.1 \mathrm{ml})$ were each randomly and blindly rubbed into a different test site using a clean finger cot, leaving one site per arm as a damaged, nontreated control. TEWL and capacitance measurements were repeated at 1, 2 and $4 \mathrm{~h}$ after treatment. Vehicle contained water, sorbitan monostearate, polyoxyl 20 cetostearyl ether, cetostearyl alcohol, mineral oil, propylparaben, methylparaben, butylated hydroxyl toluene, and hydroxyethyl cellulose and oleyl alcohol. DFD-01 was the same formulation with the addition of betamethasone dipropionate, $0.05 \%$.

Participants were required to wear nonocclusive, noncontact, protective arm guards in between product application and each of the follow-up measurements to minimize product transfer and site contact with foreign material. Participants were instructed to refrain from exercising or drinking hot beverages or cold/ iced coffee or tea during the $2 \mathrm{~h}$ prior to each measurement on day 2.

\section{Study 2: Skin Flexibility}

Study 2 enrolled healthy white males and females, aged 18-55 years. Unlike Study 1, there were no Fitzpatrick skin type restrictions. Prestudy requirements regarding skin product use and study inclusion and exclusion criteria were similar to those in Study 1. The use of arm guards between measurements and the restrictions on exercise and consumption of hot and cold drinks between readings were also similar to Study 1.

Each volunteer had six 5 by $5 \mathrm{~cm}$ sites (three sites per volar forearm) outlined (Fig. 1), and baseline cutometer measurements were taken from each site. Skin flexibility was tested with a Cutometer $^{\circledR}$ MPA $580 \quad$ (Courage + Khazaka Electronic $\mathrm{GmbH})$. In this study, the instrument

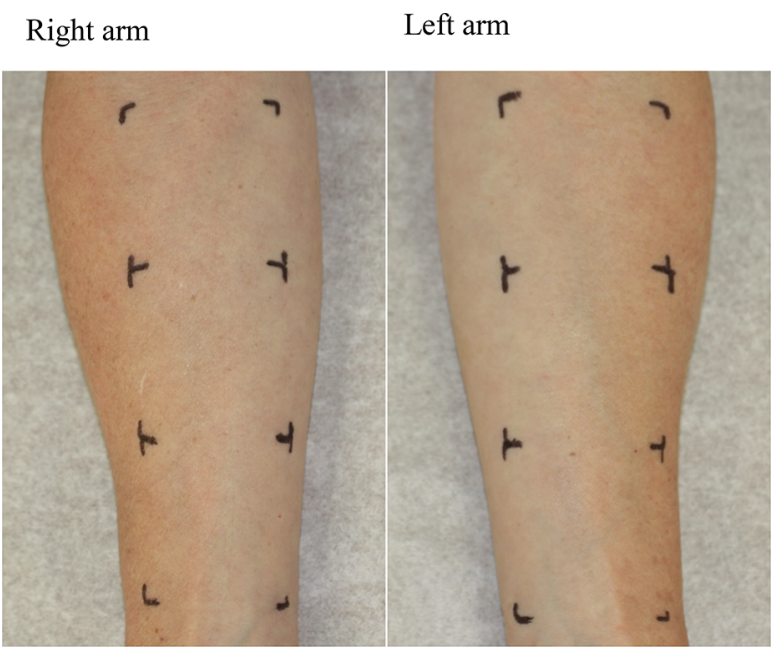

Fig. 1 Demarcated test sites on the forearm for Study 2

specifications used were a 2-mm-diameter aperture probe, mode 1 (constant negative pressure during on period), vacuum 450 mbar, $2 \mathrm{~s}$ on, $2 \mathrm{~s}$ off, three cycles. The instrument technician took one measurement (three cycles) from each site.

DFD-01 and vehicle $(0.1 \mathrm{ml})$ were each randomly and blindly rubbed into one test site on each arm using a clean finger cot; the central site on each arm remained untreated as a control. Flexibility measurements were repeated at 1,2 and $4 \mathrm{~h}$ after treatment, with the maximum extension of the third cycle (R3) used for analysis. Change in control sites subtracted from change in treated sites determined treatment effects.

\section{Compliance with Ethics Guidelines}

Ethical consent for Study 1 and Study 2 was received from IntegReview IRB prior to enrollment. For both studies, volunteers provided written informed consent and Health Insurance Portability and Accountability Act (HIPAA) authorization prior to participation. Studies were conducted in compliance with the Declaration of Helsinki, current International Council for Harmonisation guidelines for Good Clinical Practice (GCP), Food and Drug Administration (FDA) regulations and other applicable laws and regulations. 


\section{Statistics}

For both Study 1 and Study 2, repeated-measures ANOVA was used to compare the two test products and the nontreated controls. If differences were significant, multiple post hoc comparisons were made using a Tukey-Kramer multiple comparison test. For all analyses, the level of significance was $P<0.05$.

\section{RESULTS}

\section{Study 1: Transepidermal Water Loss}

Study 1 enrolled 17 female subjects with a median age of 39 years (range 21-52 years) and one male subject, aged 28 years. Dry shaving of the skin on day 1 caused elevated TEWL rates in all participants, confirming disruption of the stratum corneum barrier. On day 2, mean TEWL rates were measured at the nonshaved control sites on the side of the volar forearm with intact stratum corneum and were recorded as $7.1+1.4$ (SD) $\mathrm{g}$ water $/ \mathrm{cm}^{2}$ skin surface $/ \mathrm{h}$, which is well within the normal range; this remained constant over the course of the study.

Because there were slight differences in baseline TEWL values, the net change from baseline was calculated for each time point, and these values were analyzed for significance. TEWL rates were statistically significantly lower in shaved sites treated with either DFD-01 or vehicle compared with shaved but nontreated sites at $1 \mathrm{~h}$ after product application and for all time points thereafter $(P<0.001$; Fig. 2$)$. TEWL rates were similar between DFD-01 and vehicle throughout the study.

Skin surface hydration levels measured by capacitance readings for nonshaved control skin areas were within normal range and remained constant over the 4-h study period (Fig. 3). As expected, razor-shaved skin was drier than nonshaved skin, and capacitance was significantly decreased in all the dry shaved sites (Fig. 3). To control for slight differences in baseline values, the net change from baseline at each time point was calculated and analyzed for significance. Capacitance testing showed that both DFD-01 and vehicle were effective moisturizers when applied to the dry skin induced by dry shaving, with no significant differences between the two products at any time point. Capacitance measurements at 1, 2 and $4 \mathrm{~h}$ post treatment all showed significant differences between razor-chafed untreated skin and skin treated with either vehicle or DFD-01 $(P<0.001$, repeated-measures ANOVA and Tukey-Kramer multiple comparisons test; Fig. 3). Skin surface hydration levels of treated skin became equivalent to those of normal, intact skin. There were no adverse events during the study.

\section{Study 2: Skin Flexibility}

For this study, 15 females (mean age 41.6 years, range 23-54) and three males (mean age 34 years, range 25-51) were enrolled. Both vehicle and DFD-01 produced the net effect of an increase in R3 (maximum extension) value, indicating greater softening/flexibility of the skin. At $1 \mathrm{~h}$ post treatment, there was a highly significant difference between DFD-01 and no treatment $(P<0.01)$; overall, the effect was greater for DFD-01 than for vehicle (Fig. 4). Over time, R3 values decreased in treated skin samples, indicating firming of the skin. There were no reports of serious adverse events in this study. There was one nonserious adverse event (mild numbness of left hand), which was judged as unlikely to be related to the study products.

\section{DISCUSSION}

These studies were designed to investigate the properties of DFD-01 and its vehicle formulation on both normal, unbroken skin and dry shaved skin. Changes in TEWL and evaluation of skin moisturization and skin flexibility were compared between normal and damaged skin.

Study 1 demonstrated a reduction in TEWL with both DFD-01 and vehicle, showing that both products increased water retention in shaved skin. The capacitance investigation in Study 1 found that the level of skin hydration in razor-chafed skin treated with either DFD-01 or vehicle was similar to that of unshaved control skin samples. This suggests that these products 


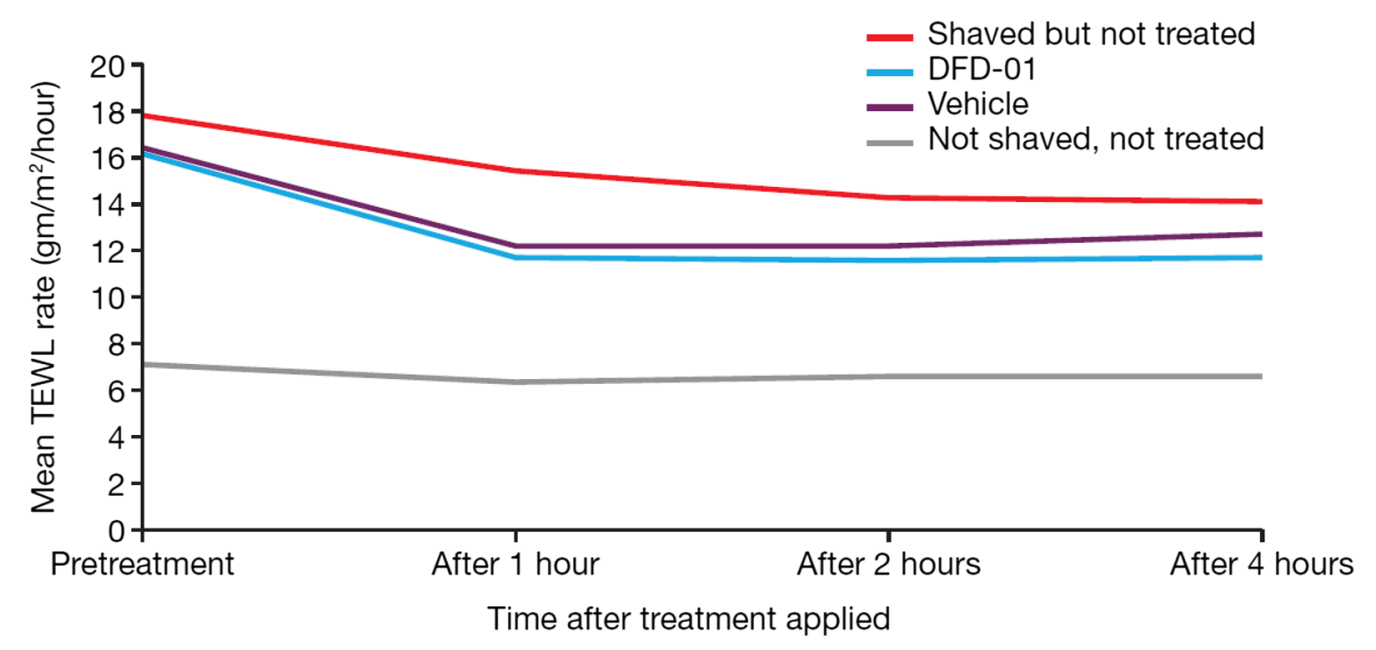

Fig. 2 Study 1, mean transepidermal water loss rate from volar forearm skin previously dry shaved and treated with DFD-01 and its non-steroid-containing vehicle, measured over the course of $4 \mathrm{~h}$. After $1 \mathrm{~h}$, the difference between shaved but not treated skin and shaved and treated with either DFD-01 or vehicle was significant for all time points $(P<0.001$, ANOVA $)$

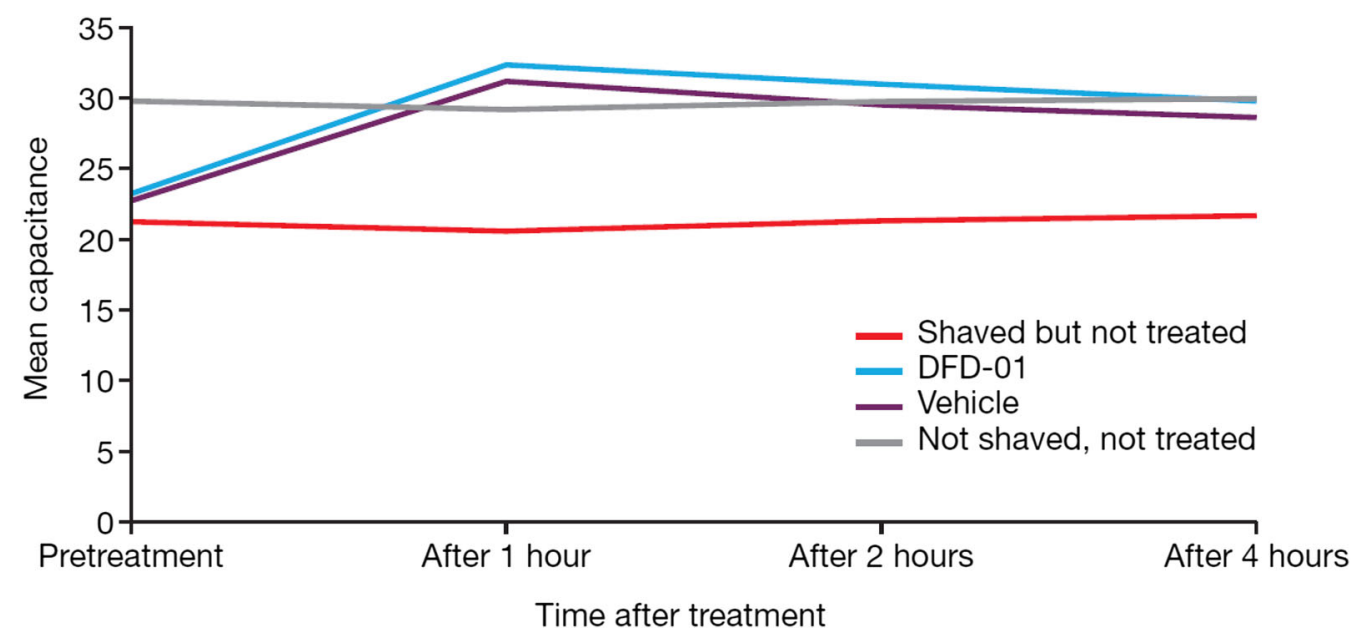

Fig. 3 Study 1, skin hydration levels of volar forearm based on Corneometer ${ }^{\circledR}$ capacitance readings of previously dryshaved skin treated with DFD-01 and its non-steroid-containing vehicle, measured over the course of $4 \mathrm{~h}$. Hydration

are effective for softening and moisturizing damaged stratum corneum.

Study 2 showed that over the course of $4 \mathrm{~h}$, normal skin treated with either DFD-01 or vehicle demonstrated a net effect of softening and increased flexibility. These findings indicate that the product has an emollient-like effect. Over the 4-h duration of Study 2, all skin sites, including the control sites, showed an increase in R3 values. measurements for unshaved skin and skin treated with either vehicle or DFD-01 were significantly greater than shaved but untreated skin at 1,2 and $4 \mathrm{~h}(P<0.001$, ANOVA, Tukey-Kramer multiple comparisons test)

This may be due to repeated suction or the continued acclimatization of the volunteers, who went from a rainy external environment to the cool, dry study environment.

The findings in Study 1 and Study 2 demonstrate that the DFD-01 vehicle helps to reduce TEWL and improve barrier function, supporting skin healing. The increase in flexibility indicates that the vehicle formulation is softening the 


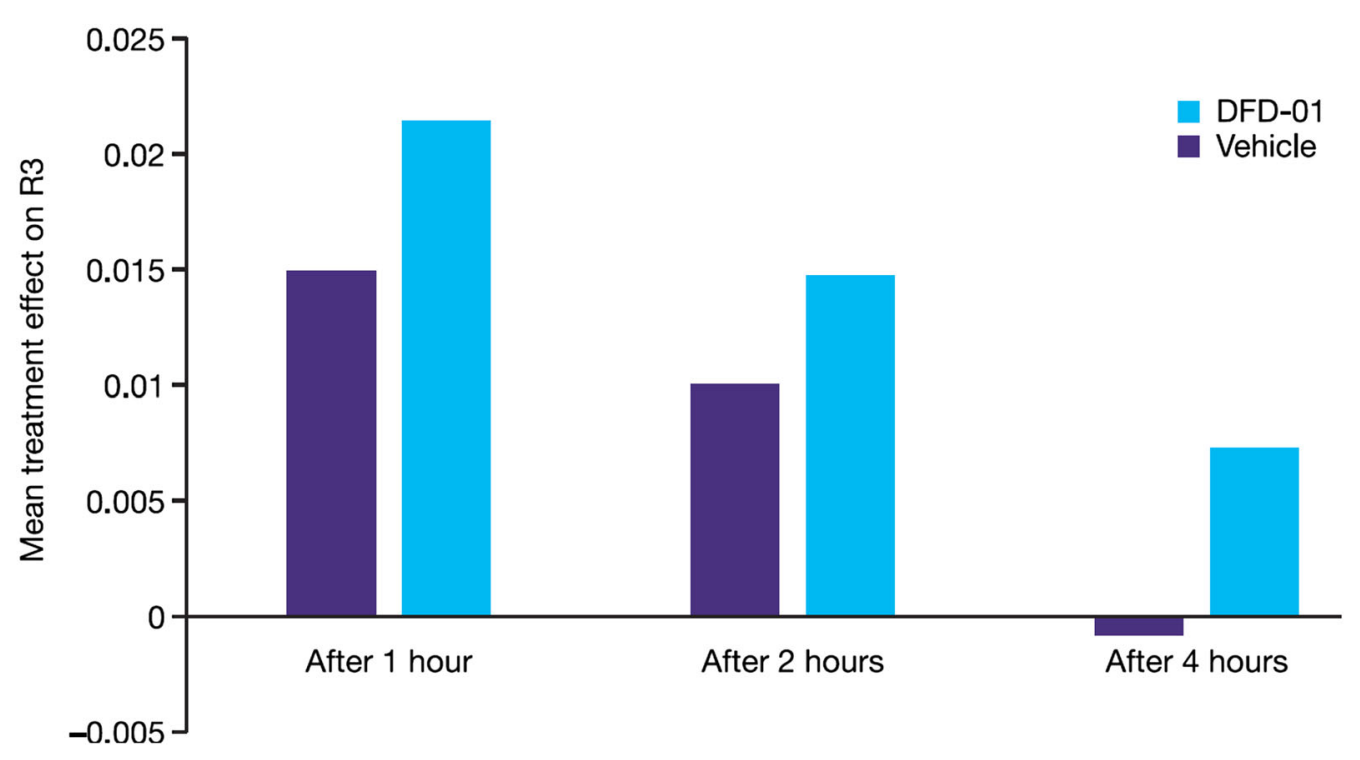

Fig. 4 Study 2, skin flexibility measurements from healthy volunteer volar forearm skin after application of DFD-01 or vehicle. The mean change in the R3 (maximum extension) cutometer measures of control sites at baseline

damaged skin and returning it to a healthier state. The similar effect seen with DFD-01 and its vehicle is expected following the single application used in these studies on healthy skin. On psoriatic skin, the additional therapeutic effect of the betamethasone dipropionate $0.05 \%$ in DFD-01 would be expected to become evident with repeated applications. The emollient properties of the vehicle demonstrated in these studies may contribute to the onset of efficacy of DFD-01. In a head-to-head comparison, DFD-01 showed rapid improvement as early as day 4 in outcomes including Total Sign Score, erythema and scaling, compared with a superpotent augmented betamethasone dipropionate $0.05 \%$ lotion (Diprolene) [10].

There were no serious adverse events reported in Study 1 or Study 2. Subjects in Study 1 reported no adverse events. One subject in Study 2 experienced an unrelated adverse event of mild hand numbness. Previous studies, in which DFD-01 was administered twice daily for 28 days, reported application site pain and pruritus as the most common adverse events [12]. The lack of side effects associated with DFD-01 or its vehicle in these studies is expected following a single exposure. was subtracted from the change in each of the treated sites to produce the treatment effect. At $1 \mathrm{~h}$, there was a significant difference between DFD-01 and no treatment $(P<0.01)$; no other significant differences were observed

Generalizability of these findings is limited by small sample size and use of nonpsoriatic skin. Studying healthy skin with standardized barrier disruption allows for appropriate control of methodologies using evaporimeter and Corneometer assessments. The inflammation present in psoriatic skin confounds the ability to evaluate barrier repair alone.

The positive role of moisturizers and emollients in patients with psoriasis is well documented $[13,14]$. These products help reduce scale and play a supportive role in normalizing hyperproliferation, differentiation and apoptosis. Improved barrier function and stratum corneum hydration make the epidermis more resistant to external stressors [15]. Reduction of superficial scale improves appearance, and softening the plaques may reduce discomfort, itch and irritation [16].

\section{CONCLUSIONS}

These two studies demonstrate that both DFD-01 and its vehicle provide moisturization, softening of razor-shaved skin and reduced TEWL compared with no treatment. 
Undamaged skin is more flexible after the application of DFD-01 or its vehicle formulation. These results support the emollient-like properties of DFD-01, which may require repeated application for continued effect.

\section{ACKNOWLEDGEMENTS}

The studies reported in this manuscript were designed and funded by the Dr. Reddy's Laboratories group of companies, including Promius Pharma. Article processing charges were funded by Dr. Reddy's Laboratories. All authors had full access to all the data in this study and take complete responsibility for the integrity of the data and accuracy of the data analysis. All named authors meet the International Committee of Medical Journal Editors (ICMJE) criteria for authorship for this manuscript, take responsibility for the integrity of the work as a whole and have given final approval to the version to be published. Editorial assistance was provided by CUBE Information, Brighton, UK, who developed the figures, provided fact checking and formatted the manuscript prior to submission. These services were funded by the Dr. Reddy's Laboratories group of companies.

Disclosures. J. Mark Jackson has received research, honoraria, consulting and/or other support from AbbVie, Amgen, Celgene, Dermira, Galderma, Janssen, Lilly, Medimetriks, Merck, Novartis, Pfizer, Promius Pharma and Top MD. Gary L. Grove served as clinical study investigator without incentives or compensation beyond normal fees. Kent Allenby is an employee of Dr. Reddy's Laboratories and owns stock in the company, but did not receive any specific funding related to this study. Tim Houser is an employee of cyberDERM and has no relevant conflicts of interest.

Compliance with Ethics Guidelines. All procedures followed were in accordance with the ethical standards of the responsible committee on human experimentation (institutional and national) and with the Helsinki Declaration of 1964, as revised in 2013.
Informed consent was obtained from all patients for being included in the study.

Data Availability. The data sets and analyses generated from these studies are available from the corresponding author upon reasonable request.

Open Access. This article is distributed under the terms of the Creative Commons Attribution-NonCommercial 4.0 International License (http://creativecommons.org/licenses/ by-nc/4.0/), which permits any noncommercial use, distribution, and reproduction in any medium, provided you give appropriate credit to the original author(s) and the source, provide a link to the Creative Commons license, and indicate if changes were made.

\section{REFERENCES}

1. Tagami H. Electrical measurement of the hydration state of the skin surface in vivo. Br J Dermatol. 2014;171(Suppl 3):29-33.

2. Menter A, Gottlieb A, Feldman SR, et al. Guidelines of care for the management of psoriasis and psoriatic arthritis. Section 1. Overview of psoriasis and guidelines of care for the treatment of psoriasis with biologics. J Am Acad Dermatol. 2008;58:826-50.

3. Prignano F, Ricceri F, Pescitelli L, Lotti T. Itch in psoriasis: epidemiology, clinical aspects and treatment options. Clin Cosmet Investig Dermatol. 2009;2:9-13.

4. Dawn A, Yosipovitch G. Treating itch in psoriasis. Dermatol Nurs. 2006;18:227-33.

5. Weisshaar E, Dalgard F. Epidemiology of itch: adding to the burden of skin morbidity. Arch Derm Venereol. 2009;89:339-50.

6. National Eczema Society. Emollients. Available at: http://www.eczema.org/emollients. Accessed January 20, 2017.

7. Correa MCM, Nebus J. Management of patients with atopic dermatitis: the role of emollient therapy. Dermatol Res Pract. 2012;2012:836931.

8. Luger T, Seite $S$, Humbert $\mathrm{P}$, et al. Recommendations for adjunctive basic skin care in patients with psoriasis. Eur J Dermatol. 2014;24:194-200. 
9. Menter A, Korman NJ, Elmets CA, et al. Guidelines of care for the management of psoriasis and psoriatic arthritis. Section 3. Guidelines of care for the management and treatment of psoriasis with topical therapies. J Am Acad Dermatol. 2009;60:643-59.

10. Fowler JF Jr, Herbert AA, Sugarman J. DFD-01, a novel, medium potency betamethasone dipropionate $0.05 \%$ emollient spray, demonstrates similar efficacy to augmented betamethasone dipropionate $0.05 \%$ lotion for the treatment of moderate plaque psoriasis. J Drugs Dermatol. 2016;15:154-62.

11. Kircik L, Bagel J. DFD-01 a novel emollient spray formulation of $0.05 \%$ betamethasone dipropionate effectively treats plaque psoriasis on knees and elbows. Poster presentation at Maui Derm, Grand Wailwa, Maui, Hawaii. January 25-29, 2016.

12. Stein Gold L, Jackson JM, Knuckles MLF, Weiss JS. Improvement in extensive moderate plaque psoriasis with a novel emollient spray formulation of betamethasone dipropionate $0.05 \%$. J Drugs Dermatol. 2016;15:300-8.
13. British Association of Dermatologists. Topical treatments for psoriasis. Revised November 2013. Available at: http://www.bad.org.uk/library-media\%5C documents\%5CPsoriasis\%20-\%20topical\%20treatments\%20Updated\%20Nov\%202013\%20-\%20lay\% 20reviewed\%20Nov\%202013.pdf. Accessed January 20, 2017.

14. American Academy of Dermatology. Psoriasis: recommendations for emollients. Available at: https://www.aad.org/practice-tools/quality-care/clinical-guidelines/psoriasis/topical-therapy/recommendations-for-emollientsaad.org. Accessed January 20, 2017.

15. Fluhr JW, Cavallotti C, Berardesca E. Emollients, moisturizers, and keratolytic agents in psoriasis. Clin Dermatol. 2008;26:380-6.

16. Psoriasis and Psoriatic Arthritis Alliance. Emollients and psoriasis. Available at: http://www.papaa.org/ further-information/emollients-and-psoriasis. Accessed January 20, 2017. 